\title{
Comparing two adaptive methods for removing cardiac beat artifact in esophageal pressure measurement: MANC and AFIR
}

\author{
Y. P. Cheng*, H. D. Wu**, G. J. Jan* \\ * Department of Electrical Engineering, National Taiwan University, Taipei 10764, Taiwan \\ ** Department of Diagno-therapeutics, National Taiwan University Hospital, Taipei 10016, Taiwan \\ *E-mail: d82056@cctwin.ee.ntu.edu.tw
}

\begin{abstract}
The measurement of esophageal pressure $(\mathrm{Pes})$ is a simple technique that can be used to calculate the lung mechanical parameters. In clinical, the traditional strategy for cardiac beat artifact minimization in Pes measurement is to adjust either the position of esophageal balloon or the posture of the body under test. We proposed a new scheme of modified adaptive noise cancellation (MANC) method and adaptive FIR (AFIR) filter to eliminate the cardiac beat artifact from Pes measurement. In experiments, we also find the harmonic effect to interfere the measurement of Pes. To use the MANC which we do not have to carefully adjust the position of esophageal balloon to avoid the results being affected by body posture and it is independent with frequencies variation of cardiac beat and respiration. To use the AFIR, which is suitable for most case that the frequency of cardiac beat is apart from the respiration frequency but not suitable for the frequencies or harmonic are overlap slightly.
\end{abstract}

Keyword: adaptive filter, adaptive noise cancellation, FIR, esophageal pressure, LMS.

\section{Introduction}

Since the measurement of $\mathrm{Pip}$ has potential risks for testees, another method that applied esophageal balloon for indirect determination of intrapleural pressure was proposed in 1959 [1]. The cardiac beat artifact in the measurement of Pes can be reduced but can't be removed completely by adjusting the balloon positions or postures of patients [1],[2]. In this research, we compared two adaptive methods, MANC [3] and AFIR filter [4] has been used to remove the cardiac beat interference from $P$ es.

\section{Methods}

The diagram of this research is shown in Fig. 1. We measured Pes signal of Brown-Norway rats while off-line processing was done with MANC and AFIR.

The functional block diagram of MANC is shown in Fig. 2. The MANC is based on FIR Wiener filter structure and using least mean squared (LMS) algorithm to adapt the weights which are coefficients of filter [3],[5],[6],[7]. The important discrepancy between MANC and ANC is input signals. The functional block diagram of AFIR is shown in
Fig. 3 which is used a spectrum analyzer and a coefficient look-up table (CLT) to find the cut-off frequency. In AFIR, the filter technique is using Kaiser window [4] to design a multiple cut-off frequency (MCF) band-stop filter and using Hamming window to design a low-pass filter (LPF).

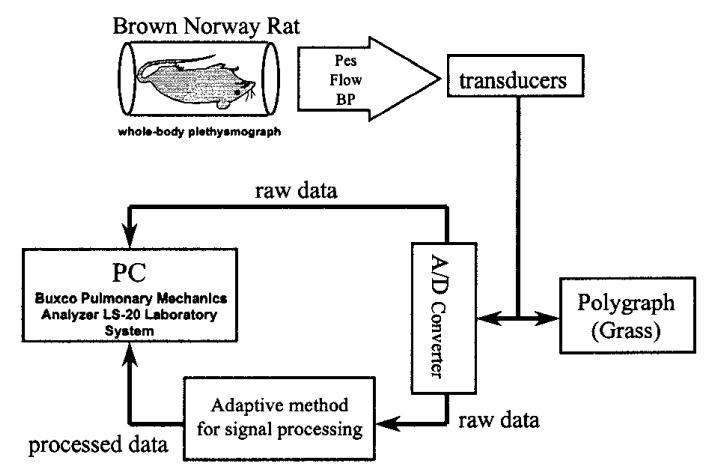

Fig. 1 Functional block diagram of the system.

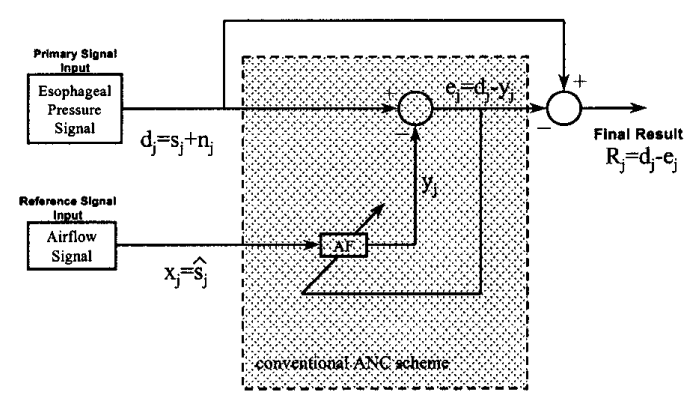

Fig. 2 Functional block diagram of modified ANC (MANC), in which the shaded line area is the conventional ANC structure.

\section{Results}

We carried out six experiments and the results are used to evaluate the performance of two adaptive methods. In 
experiment 1 and 2, we inserted the catheter into esophagus without careful adjustment, and Pes signal were seriously interfered by cardiac pulsation. In experiment 3 , the catheter was adjusted carefully to minimize the cardiac beat artifact. And in experiment 4, the rat was placed in right lateral decubitus position. Two experiments $(5$ and 6$)$ were performed in different respiratory frequency conditions of an identical rat (but a different one from that of experiment 1-4). The change of respiratory frequency was induced by bronchoconstrictor. Using the MANC, the LMS algorithm can adjust the filter weights to optimum and result is shown in Fig. 4. Figure 4 is the result of experiment 6 , the results of experiment 1-6 are alike which their filtered signal are immune from artifact and we find the characteristic of filter is adjusted automatically by input signals.

A simple LPF is designed by using Hamming window (we called Hamming LPF) and the result of experiment 6 is shown in Fig. 5. In Fig. 5, the cut-off frequency is $3.5 \mathrm{~Hz}$ and the order of filter is 210 [4]. We find that if the cutoff frequency is selected between $1^{\text {st }}$ harmonic of Pes signal and cardiac beat main frequency, the results are similar as which of MANC. But these case are only suitable for experiment 1 4 , for experiment 5 and 6 are not suitable because of frequencies are nearly or overlap slightly which to select the cutoff frequency is difficulty.

Figure 6 shows the result of AFIR which using MCF designed by Kaiser window. The purpose of MCF bandstop filter is designed to eliminate cardiac beat signal and its harmonics. As same as Hamming LPF, the results of experiment $1-4$ are better than experiment 5 and 6 but not quite well comparing with MANC. In Fig. 6(d), the filtered Pes signal is similar as the original Pes signal. Same result is found in experiment 5 .

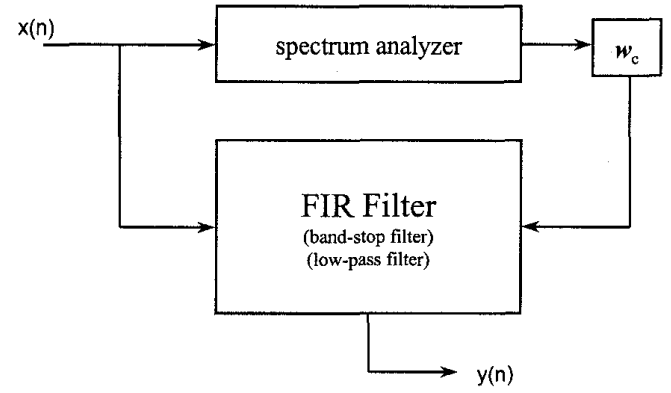

Fig. 3 Functional block diagram of adaptive FIR (AFIR) filter. (a)
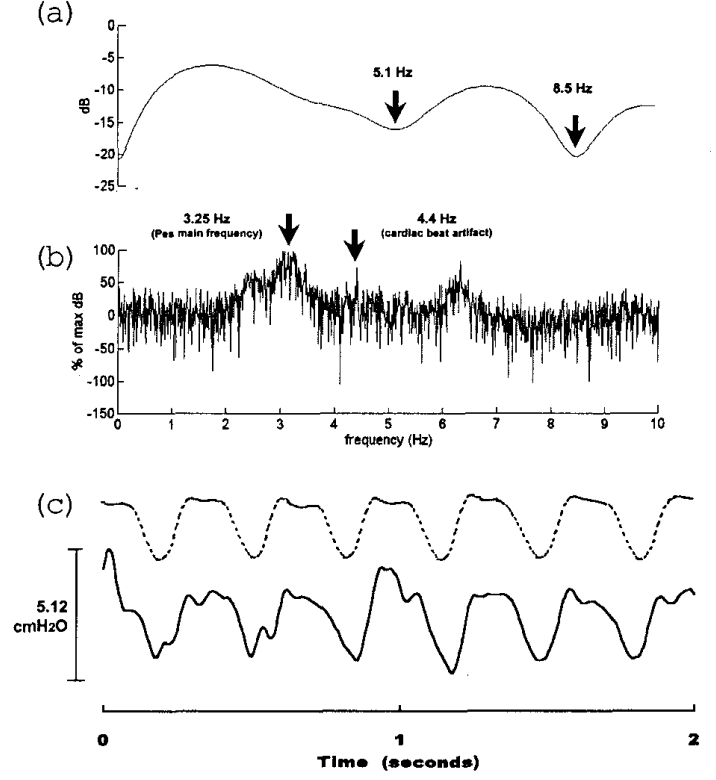

Fig. 4 Result of experiment 6. (a) The frequency response curve of the filter in MANC. (b) Power spectrum density of original Pes signal. (c) The relationship of filtered and original signals, upper line is filtered Pes signal and lower line is original Pes signal.
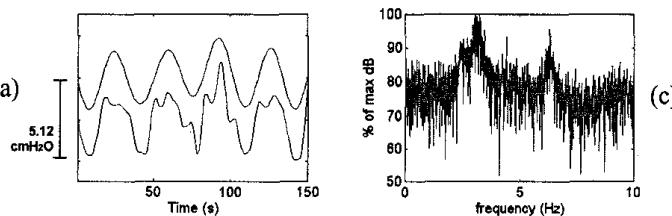

(b)
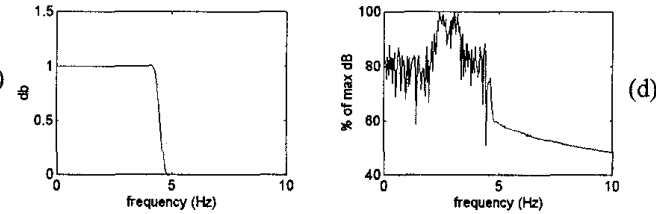

Fig. 5 The results of AFIR by Hamming LPF in experiment 6 , order $=210$ and cut-off frequency $w_{\mathrm{c}}=3.5 \mathrm{~Hz}$. (a) The relationship of filtered and original signals, upper line is filtered Pes signal and lower line is original Pes signal. (b) The frequency response curve of the LPF. (c) Power spectrum density of original Pes signal. (d) Power spectrum density of filtered Pes signal. 
(a)

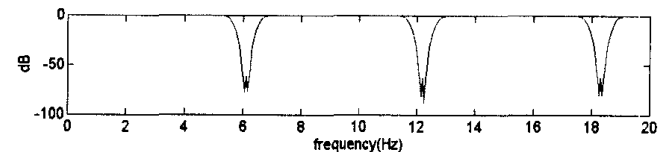

(b)

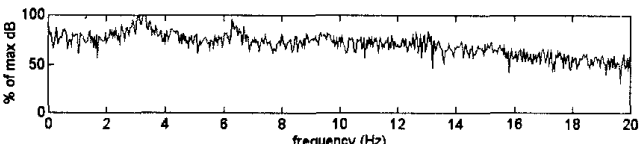

(c)

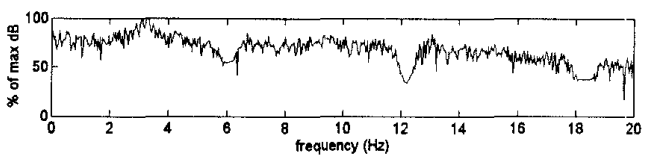

(d)

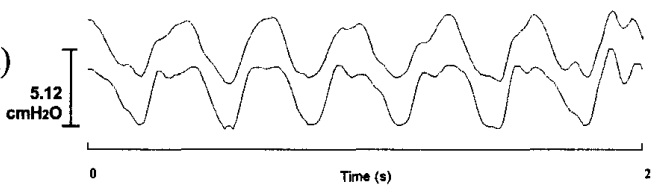

Fig. 6 The results of AFIR by Kaiser MCF bandstop filter in experiment 6. (a) Frequency response curve of filter, order $=500$ and $A s=60$. (b) Power spectrum density of original Pes signal. (c) Power spectrum density of filtered Pes signal. (d) The relationship of filtered and original signals, upper line is filtered Pes signal and lower line is original Pes signal.

\section{Discussion}

There is a very important phenomenon, the harmonic effect. In normal case or the cardiac beat frequency is far away from the respiration frequency, the performance of AFIR and MANC is similar. But for the case of frequencies are nearly or slightly overlap, the MANC is also provide quit well performance because of the MANC is independent of frequencies variation (Fig. 4). However, AFIR is not suitable to apply in these conditions. For example, in Fig. 5, it's easily seen that the filtered signal is similar to a sinusoidal waveform. But in real sense, Pes signal should contain an end-exhalation plateau. We predict that the plateau may be performed by high frequency that is harmonic components. Using a simple LPF would cut all high-frequency components including harmonics. If we remain $1^{\text {st }}$ harmonic of Pes signal and cutoff all high frequencies including cardiac beat frequency, we can obtain well results are similar as which of MANC. We use MCF bandstop filter to eliminate the artifact (Fig. 6), the performance is not well because frequencies of cardiac beat and respiration frequency are nearly and harmonic is overlap slightly. The MANC has been verified that it is good processed results under the change condition of respiratory and heart beat frequency.

\section{Conclusions}

Comparing two methods, if we use the spectrum analyzer, we must add the FFT algorithm and to define the frequency range to find cut-off frequency which decides the coefficient of filter. Because the respiratory and cardiac frequencies are not fixed at any time, the sampling rate and data interval for processing must be considered. Furthermore, many parameters of filter, such as passband edge frequency, stopband edge frequency, peak passband deviation and stopband deviation could be defined. The LMS algorithm can automatically adjust the filter weights for the passing signal frequency band by input signal feature, which is simpler and more easily implemented by a DSP processor such as the TMS320C series processor. For these reasons, we prefer to choosing the MANC and assure its feasibility in this research.

\section{Acknowledgment}

We would like to acknowledge the National Science Council of the Republic of China to support this research under grant contract No. NSC-86-2213-E-002-060. and NSC-87-2213-E002-082.

\section{References}

[1] J. H Knowles, S. K. Hong, and H. Rahn, "Possible error using esophageal balloon in determination of pressurevolume characteristics of the lung and thoracic cage." $J$. Appl. Physiol. 14(4), 525-530, 1959.

[2] J. Milic-Emili, J. Mead, and J. M. Turner, "Topography of esophageal pressure as a function of posture in man." J. Appl. Physiol. 19(2), 212-216, 1964.

[3] Y. P. Cheng, H. D. Wu, G. J. Jan and C. Y. Wang, "Adaptive method for removing cardiac beat artifact in esophageal pressure measurement", Compu. \& Biomed. Res., revised.

[4] J. Ye, and T. T. C. Choy, "On-line respiratory artefact removal via adaptive FIR filters in rheopneumographic measurement." Med. Biol. Eng. Comput. 32, 620-624, 1994.

[5] E. C. Ifeachor, and B. W. Jervis, Digital Signal Processing, A Practical Approach, Addison-Wesley, Wokingham, 1993.

[6] S. V. Vaseghi, Advanced Signal Processing and Digital Noise Reduction, Wiley, New York, 1996.

[7] B. Widrow, J. R. Glover, J. M. McCool, J. Kaunitz, C. S. Williams, R. H. Hearn, J. R. Zeidler, E. Dong, and R. C. Goodlin, "Adaptive noise cancelling: principles and applications", Proc. IEEE 63, 1692-1716, 1975. 